\title{
Instrumento para avaliar a combinação de alimentos para tornar o ferro mais biodisponível na dieta
}

\author{
Instrument to evaluate the combination of foods \\ to make iron more bioavailable in the diet
}

Haydée Serrão Lanzillotti ${ }^{1}$

Maria Elisa Barros ${ }^{1}$

Fernanda da Motta Afonso ${ }^{1}$

Roseane Moreira Sampaio Barbosa ${ }^{2}$

${ }^{1}$ Instituto de Nutrição, Universidade do Estado do Rio de Janeiro. R. São Francisco Xavier 280 a

526 lado par, Maracanã. 20550-013 Rio de Janeiro RJ Brasil.

haydeelan@gmail.com

${ }^{2}$ Departamento de Nutrição

Social, Faculdade de

Nutrição, Universidade

Federal Fluminense. Niterói

RJ Brasil.
Abstract The scope of this research was to develop an instrument to evaluate the knowledge which adult women have of food combinations in order to make iron bioavailable in the diet of children. An exploratory factor analysis study (Likert scale) was conducted applying a questionnaire on a sample comprising 151 women and then applying Principal Factor Analysis and Likelihood Ratio Analysis. The Kaiser-Meyer-Olkin (KMO) test, the Doornik-Hansen test, the Kaiser Guttman criterion and the correlation between factors and theoretical assumptions were applied. Reliability was assessed by Cronbach's alpha and composite reliability. Total KMO (0.5942) showed the adequacy of the sample. The Doornik-Hansen test showed multivariate normality of the scores observed. Factorial loads, error variance of items and correlation between factors pointed to the two-dimensional nature of the questionnaire, which decreased from 30 to 15 items. Total Cronbach's alpha was 0.8018 (95\% CI $=0.7429$ to 0.8606 ) and composite reliability (Factor $1=$ 0.3125 and Factor $2=0.3263$ ). Convergent and discriminant factorial validity revealed acceptable results. A decision was taken to reduce the instrument (from 30 to 15 items) with sufficient reliability and validity that enable it to qualify for further confirmatory factor analysis procedures.

Key words Instrument, Bioavailability, Iron, Food, Child
Resumo O objetivo foi desenvolver um instrumento para avaliar o conhecimento de mulheres adultas sobre a combinação de alimentos de forma a tornar o ferro biodisponivel na dieta infantil. Estudo fatorial exploratório de questionário (escala Likert) utilizando-se Análise de Fator Principal e estimador de razão de verossimilhança. Aplicaram-se teste KMO, teste Doornik-Hansen, critério de Kaiser Guttman, correlação entre fatores e pressupostos teóricos. Confiabilidade avaliada pelo Alfa de Cronbach e confiabilidade composta. Participaram do estudo 151 mulheres. KMO total $(0,5942)$ mostrou adequação da amostra. Teste Doornik-Hansen atestou normalidade multivariada dos escores observados. Cargas fatoriais, variância do erro dos itens e correlação entre os fatores apontaram para a bidimensionalidade do questionário, que passou de 30 para 15 itens. Alfa de Cronbach total de 0,8018 (95\% IC = 0,7429 a 0,8606) e confiabilidade composta (Fator $1=$ $0,3125$ e Fator $2=0,3263)$. Validade fatorial convergente e discriminante apresentaram resultados aceitáveis. Concluiu-se por redução de instrumento validado (30 para 15 itens) com confiabilidade e validade que o habilita para posterior procedimento de análise fatorial confirmatória.

Palavras-chave Instrumento, Biodisponibilidade, Ferro, Alimentos, Criança 


\section{Introdução}

No Brasil, até o momento, não há levantamento nacional sobre a prevalência de anemia ferropriva. Dados regionais têm demonstrado elevada prevalência desta anemia em todas as idades e níveis socioeconômicos ${ }^{1}$. A revisão sistemática realizada por Jordão et al. ${ }^{2}$ mostrou prevalência de anemia ferropriva de $53 \%$ (em dados medianos) entre crianças menores de cinco anos de idade.

Neste cenário, na maioria das vezes, é a mãe quem modela os hábitos alimentares de seus filhos. Rotemberg e Vargas ${ }^{3}$ ao realizarem entrevistas com mães brasileiras de uma unidade municipal básica de saúde na zona sul do município do Rio de Janeiro, RJ, Brasil, identificaram que as práticas alimentares maternas estão influenciadas por hábitos urbanos de consumo.

Particularmente, no caso da anemia ferropriva em mulheres adultas, gestantes ou não, grupos considerados vulneráveis ${ }^{4}$, acredita-se ser relevante flagrar o conhecimento das mulheres sobre o modo de combinar alimentos a fim de tornar o ferro mais biodisponível na dieta. A quantidade absorvida de ferro pelo organismo pode ser modificada por componentes estimuladores e inibidores presentes nos alimentos ingeridos na mesma refeição. Os componentes estimuladores da absorção do ferro são o ácido ascórbico e o tecido muscular dos alimentos de origem animal ${ }^{5}$. A carne bovina possui 50\% do seu teor de ferro na forma de ferro heme, cuja disponibilidade varia de 15 a $35 \%{ }^{6}$. Os componentes inibidores de sua absorção são o cálcio (leite e derivados) e substâncias alimentares tais como ácido fítico-pentafosfato IP5 (trigo integral e multimisturas), ácido oxálico (espinafre, carambola, grãos integrais de arroz, trigo, centeio, aveia, cevada, milho), cafeína (café, chás mate verde e preto, guaraná, refrigerantes, bebidas energéticas e achocolatados) ${ }^{7,8}$.

Neste sentido, cresce a necessidade de se elaborar proposta metodológica que envolva instrumentos de mensuração comportamental para avaliar o conhecimento dos indivíduos sobre a combinação de alimentos de forma a biodisponibilizar o ferro na dieta. O instrumento mapear certas combinações de alimentos, as quais favorecem ou inibem a absorção de ferro, podendo contribuir para a eficácia das intervenções nutricionais na anemia ferropriva. Parte-se da hipótese de que a maioria das mulheres não está familiarizada com a combinação de alimentos, na refeição, de forma a possibilitar o aumento da biodisponibilidade do ferro na dieta.
A importância de buscar instrumentos que permitam avaliar o conhecimento dos indivíduos sobre a maneira de combinar os alimentos que contêm ferro, a fim de aumentar sua biodisponibilidade na dieta, corresponde a uma estratégia simples e imediata que vem contribuir para ações em Saúde Pública.

O objetivo do estudo foi desenvolver um instrumento para avaliar o conhecimento de mulheres adultas sobre a combinação de alimentos de forma a tornar o ferro mais biodisponível na dieta infantil.

\section{Método}

Este estudo faz parte do projeto "Nutri Fuzzy Orixás: biodisponibilidade de nutrientes sob a ótica dos sistemas baseados em Conhecimentos Orixás e Sistemas Lógicos Fuzzi", aprovado pelo Comitê em Pesquisa do Hospital Pedro Ernesto da Universidade do Estado do Rio de Janeiro, sob o protocolo número 794/2003-CEP/HUPE, cujo escopo é verificar a interação entre os nutrientes e nutrientes e fatores antinutricionais, os quais participam do sistema complexo referente à biodisponibilidade de nutrientes.

Trata-se de um estudo transversal, com 151 mulheres adultas, por amostragem acidental ${ }^{9}$ realizado em salas de espera de duas unidades ambulatoriais da Universidade do Estado do Rio de Janeiro, na cidade do Rio de Janeiro, RJ, Brasil, no período de março de 2013 a junho de 2015. A razão da escolha por essa população de interesse está afeita à maior suscetibilidade de desenvolver anemia ferropriva nesse gênero ${ }^{6}$. As mulheres adultas que desejaram participar do estudo, após conhecer seus objetivos, assinaram o Termo de Consentimento Livre e Esclarecido. A coleta de dados foi realizada por acadêmicos de Nutrição, devidamente treinados.

$\mathrm{O}$ instrumento de mensuração consta de um questionário em escala Likert ${ }^{9}$, tendo cinco opções de respostas, de direção positiva ${ }^{10}$ : discordo muito (1), discordo (2), nem discordo nem concordo (3), concordo (4) e concordo muito (5). Cada indivíduo só poderia indicar apenas uma das cinco alternativas de resposta. O desenho dos itens teve inicio com a formação de um banco de 30 itens, criado a partir da literatura especializada, Protótipo 1 (Quadro 1). Selltiz et al. ${ }^{9}$, fundamentado na escala Thurstone, sugerindo um questionário com aproximadamente 20 itens. Os itens foram criados segundo duas dimensões teoricamente pressupostas, incluindo alimentos 
Quadro 1. Protótipo do instrumento para avaliar o conhecimento de mulheres adultas sobre a combinação de alimentos para tornar o ferro mais biodisponível na dieta infantil.

\begin{tabular}{|c|c|}
\hline Item & Descrição do item \\
\hline i1- I & $\begin{array}{l}\text { Não cozinhar beterraba no feijão, porque a beterraba contém substâncias que prejudicam a absorção do } \\
\text { ferro do feijão. }\end{array}$ \\
\hline $\mathrm{i} 2-\mathrm{E}$ & $\begin{array}{l}\text { Sempre dar na sobremesa uma fruta, pois contém vitamina C que ajuda na absorção do ferro no } \\
\text { organismo. }\end{array}$ \\
\hline $\mathrm{i} 3-\mathrm{E}$ & Tomar suplemento de ferro junto com suco de laranja para que o ferro seja mais absorvido. \\
\hline i4-E & $\begin{array}{l}\text { Consumir melado com alguma fruta, pois sei que ele é rico em ferro e junto com a vitamina } \mathrm{C} \text { da fruta } \\
\text { ele se torna melhor absorvido. }\end{array}$ \\
\hline i5-I & $\begin{array}{l}\text { Não consumir mate gelado no almoço e jantar por conter substâncias que dificultam a absorção do } \\
\text { ferro dos alimentos. }\end{array}$ \\
\hline i6-E & Comer fígado uma vez por semana, pois ele é rico em ferro. \\
\hline $\mathrm{i} 7-\mathrm{E}$ & Todo dia comer carne, frango ou peixe, pois sei que são as melhores fontes de ferro. \\
\hline i8-I & $\begin{array}{l}\text { Não comer iogurte após o almoço e jantar, pois o cálcio presente neste alimento compete com o ferro } \\
\text { contido nos legumes e verduras. }\end{array}$ \\
\hline i9-I & Não comer alimentos ricos em cálcio, como leite e queijos, antes e nem após as grandes refeições. \\
\hline i10-I & Deixar o feijão de remolho antes de seu preparo para que o ferro do feijão seja mais bem absorvido. \\
\hline i11-E & $\begin{array}{l}\text { Comer os vegetais junto com a carne, pois o ferro contido na carne ajuda na absorção do ferro contido } \\
\text { nos vegetais, mas não se esquecer de comer frutas na sobremesa. }\end{array}$ \\
\hline i12-E & $\begin{array}{l}\text { No momento que não tiver carne, frango ou peixe, comer ovo junto com suco de fruta para melhorar a } \\
\text { absorção de ferro do ovo. }\end{array}$ \\
\hline i13-E & Cozinhar os alimentos em panela de ferro porque aumenta a quantidade de ferro neles. \\
\hline i14-I & $\begin{array}{l}\text { Não colocar ovo no espinafre porque o espinafre contém substâncias que dificultam a absorção do ferro } \\
\text { do ovo. }\end{array}$ \\
\hline i15-I & $\begin{array}{l}\text { Não comer pudim de leite como sobremesa porque o cálcio presente atrapalha a absorção do ferro da } \\
\text { refeição. }\end{array}$ \\
\hline i16-I & Não beber café depois do almoço ou jantar, porque ele irá atrapalhar a absorção do ferro. \\
\hline i17-E & Comer verduras verdes na dieta, porque elas são ricas em ferro. \\
\hline i18-E & Comer frutas como sobremesa, porque elas melhoram a absorção do ferro da dieta \\
\hline i19-E & $\begin{array}{l}\text { Comer carne, frango, peixe ou ovo duas vezes ao dia (almoço e jantar) com o objetivo de reduzir o risco } \\
\text { de anemia. }\end{array}$ \\
\hline i20-E & Comer vísceras como, por exemplo, fígado de boi ou galinha, para evitar anemia. \\
\hline i21-I & $\begin{array}{l}\text { Não beber leite ou derivados de leite como sobremesa no almoço e jantar para não prejudicar a } \\
\text { absorção do ferro. }\end{array}$ \\
\hline i22-E & Comer carnes e vísceras, pois elas contribuem para a prevenção da anemia. \\
\hline I23-E & Aumentar o consumo de frutas ricas em vitamina C para melhorar a absorção de ferro nas refeições. \\
\hline $\mathrm{i} 24-\mathrm{E}$ & $\begin{array}{l}\text { Comer carnes em geral porque, além de fornecer ferro à dieta, ajudam a absorver o ferro presente no } \\
\text { feijão e em outros alimentos. }\end{array}$ \\
\hline i25-E & Não beber chá, mate e café durante e logo após as refeições para não prejudicar a absorção de ferro. \\
\hline i26-E & Introduzir alimentos ricos em ferro na dieta de crianças, porque a criança fica mais vulnerável a anemia. \\
\hline i27-I & $\begin{array}{l}\text { Fornecer em quantidades adequadas os vegetais verde-escuros, porque eles, em excesso, podem } \\
\text { prejudicar a absorção de ferro. }\end{array}$ \\
\hline i28-E & $\begin{array}{l}\text { Beber sucos de frutas cítricas como laranja, acerola e limão, pois, estes são ricos em vitamina C e } \\
\text { ajudam a melhorar a absorção do ferro do almoço e jantar. }\end{array}$ \\
\hline i29-I & Não beber refrigerantes, mate ou café, pois estes podem reduzir a absorção do ferro da dieta. \\
\hline $\mathrm{i} 30-\mathrm{E}$ & Alterar a dieta, introduzindo carnes, vísceras, legumes, verduras e frutas cítricas para evitar anemia. \\
\hline
\end{tabular}

E: Estimuladores da absorção do ferro =18 itens; I: Inibidores da absorção do ferro =12 itens.

estimuladores e alimentos inibidores da absorção de ferro ${ }^{6}$. O intuito do estudo foi indicar se a participante seria capaz de identificar, na redação de cada item, a combinação de alimentos que tornasse o ferro mais biodisponível na dieta.
A análise dos dados utilizou a Análise Fatorial Exploratória $(\mathrm{AFE})^{11-13}$, na qual os fatores representam as dimensões latentes do construto ${ }^{12}$. O teste Kaiser-Meyer-Olkin (KMO) verificou a adequação da amostra, utilizando-se como ponto de 
corte $0,50^{14}$, e o teste Doornik-Hansen permitiu verificar a normalidade multivariada dos escores dados aos itens do Protótipo $1^{15}$.

$\mathrm{Na}$ AFE, a extração de fatores foi realizada pelo método de Análise de Fator Principal (AFP), sem limitação do número de fatores a serem retidos, utilizando o estimador de razão de verossimilhança - likelihood ratio (LR) e, em seguida, rotação oblíqua Promax. O critério de Kaiser Guttman (fatores com autovalor $>1$ ) e os pressupostos teóricos possibilitaram desvelar a dimensionalidade do questionário ${ }^{11}$, tendo como construto "o conhecimento sobre a combinação de alimentos para tornar o ferro mais biodisponível na dieta".

Na AFP serão inferidos como itens satisfatórios os que apresentarem cargas fatoriais $(\lambda)$ maior do que 0,40 . Serão inferidos como itens com cargas cruzadas aqueles cujas cargas fatoriais, similares em dois ou mais fatores em um mesmo item, apresentem diferença entre as cargas menor do que $0,10^{16}$. A variância única (uniqueness), variância do erro, foi considerada adequada quando a magnitude se apresenta menor ou igual a $0,5^{17}$. As comunalidades, proporção de variância comum dentro de um item, foram vistas em termos de proporção da variância explicada por fatores subjacentes ${ }^{13}$.

Ao inferir sobre a dimensionalidade do questionário, foi considerada a correlação entre os fatores. Se a correlação entre os fatores (dimensões) se apresentar baixa $(<0,30)$, de direção positiva, indicará a possibilidade de dimensões de significados diferentes. No caso de a correlação entre os fatores ser negativa, sugere-se que o fator não seja retirado. Se a correlação entre os fatores se apresentar baixa/positiva ou baixa/negativa, isso indica que o conteúdo de alguns itens do questionário não são discriminadores ${ }^{11}$, ou seja, não estão distinguindo satisfatoriamente fatores estimuladores e inibidores da absorção de ferro.

$\mathrm{O}$ alfa de Cronbach, dado por:

$\alpha=k / k-1\left(\Sigma S i^{2} / S t^{2}\right)$

Onde:

$k=$ número de itens

$S i=$ variância do instrumento

$S t^{2}=$ variância da soma dos itens.

aquilatou a confiabilidade do questionário (consistência interna) e a estimativa do seu intervalo de confiança (95\%) foi realizada pelo procedimento bootstrap com 5000 reposições. Streiner e Norman ${ }^{10}$ recomendam avaliar a confiabilidade pelo limite inferior e Pasquali ${ }^{18}$ considera valores em torno de 0,70 como satisfatórios.
Embora o coeficiente alfa de Cronbach ${ }^{19}$ seja o critério mais utilizado para medir a confiabilidade de instrumentos de mensuração, Brown ${ }^{11}$ critica seu uso alegando que o coeficiente alfa de Cronbach tende a subestimar a confiabilidade. Enquanto o coeficiente alfa de Conbrach é calculado a partir da variância dos itens e da variância do instrumento, o cálculo da confiabilidade composta utiliza as cargas fatoriais e a variância do erro, uma opção mais parcimoniosa com a análise fatorial.

Neste sentido, a confiabilidade composta (CC $)^{11}$ também foi calculada para estimar a confiabilidade do instrumento. O resultado da CC é dado por:

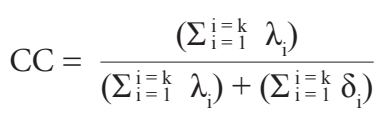

Onde:

$\lambda_{i}$ são cargas fatoriais e $\delta_{i}$ variância do erro para $k$ itens. A CC varia de 0 a 1 sendo considerada satisfatória, quando $\geq 0,70^{12}$.

A validade fatorial convergente foi avaliada pela variância da média extraída $(\mathrm{VME})^{20,21}$. A VME avalia a quantidade de variância capturada por um fator comum em relação à quantidade de variância devido às medições de erros aleatórios $^{12}$. A VME varia de 0 a 1 , e os valores de VME $\geq 0,50$ sugerem que os itens compartilham de uma elevada variância em comum $^{22}$, o que revela que pelo menos $50 \%$ da variância em uma medida é devido à variável latente. A validade fatorial discriminante (VFD) é dada pela raiz quadrada da VME. Se a raiz quadrada da VME de um fator for maior do que as correlações entre ele e os demais fatores, corrobora-se a referida validade ${ }^{12}$.

\section{Resultados}

O questionário foi respondido por 151 mulheres adultas. A matriz de entrada dos escores dados pelos respondentes apresentou, no Protótipo 1 (30 itens), valor de KMO total de 0,5942, demonstrando adequação da amostra e o teste Doornik-Hansen atestou normalidade multivariada desses escores $\left(\operatorname{chi}^{2}(60)=1055,855\right.$; Prob $>\mathrm{chi}^{2}=$ 0.0000 ) para (Tabela 1). Embora o teste KMO total apresentasse valor que permitisse considerar o tamanho da amostra adequada para estudos fatoriais, oito itens apresentaram valores abaixo do esperado (Tabela 1). Esses itens foram retirados do questionário, que apresentou novo perfil, chamado de Protótipo 2, com 22 itens (Tabela 1). 
No Protótipo 2, a repetição do cálculo do KMO mostrou que a maioria dos itens apresentou valores mais elevados dos itens de per si, quando comparados aos valores do Protótipo 1. O mesmo ocorreu em relação ao valor do KMO total $(0,7533)$. O teste Doornik-Hansen também atestou normalidade multivariada dos escores de seus itens (Tabela 1 ).

Foram criados três modelos: AFP com constrangimento por dois fatores Rotação Promax (Protótipo 1, 30 itens), AFP sem constrangimento, sem rotação (Protótipo 2, 22 itens) e AFP com constrangimento por dois fatores e rotação Promax (Protótipo 3, 15 itens). Embora o modelo do Protótipo 1 apresentasse autovalores (eigenvalue) expressivos para os dois fatores e variância explicada acumulada distribuída equitativamente ( $\mathrm{Ta}-$ bela 2), o teste KMO já havia indicado a retirada de oito itens, tendo em vista que esses apresentaram valores de KMO abaixo de 0,50 (Tabela 1).

O Protótipo 2, submetido à AFP sem constrangimento e sem rotação apresentou autovalores (eigenvalue) que indicam bidimensionalidade (Fator $1=4,5433$ e Fator $2=2,2207$ ) e a proporção da variância acumulada explicando $71,58 \%$ da latência do construto (Tabela 2). Neste modelo, aparece o Fator 3 com autovalor de 0,9991 e variância explicada de $10,57 \%$, levando a crer
Tabela 2. Modelos de Análise de Fator Principal (AFP), autovalores e proporção da variância explicada acumulada, dos protótipos do instrumento sobre a combinação de alimentos para tornar o ferro mais biodisponível na dieta infantil. Rio de Janeiro (RJ), março de 2013 a junho de 2015.

AFP constrangido a dois fatores Rotação Promax $\left({ }^{\star}\right)$ Protótipo 1 (30 itens)

\begin{tabular}{|c|c|c|}
\hline Fator & Autovalor & $\begin{array}{l}\text { Proporção da variância } \\
\text { explicada acumulada }\end{array}$ \\
\hline 1 & 4,1885 & 0,5878 \\
\hline 2 & 3,6389 & 0,5107 \\
\hline \multicolumn{3}{|c|}{$\begin{array}{l}\left.\text { AFP sem constrangimento, sem rotação }{ }^{* *}\right) \\
\text { Protótipo } 2(22 \text { itens })\end{array}$} \\
\hline Fator & Autovalor & $\begin{array}{l}\text { Proporção da variância } \\
\text { explicada acumulada }\end{array}$ \\
\hline 1 & 4,5433 & 0,4808 \\
\hline 2 & 2,2207 & 0,7158 \\
\hline 3 & 0,9991 & 0,8215 \\
\hline \multicolumn{3}{|c|}{$\begin{array}{l}\text { AFP constrangido a dois fatores e rotação Promax }(* * *) \\
\text { Protótipo } 3 \text { (15 itens) }\end{array}$} \\
\hline Fator & Autovalor & $\begin{array}{l}\text { Proporção da variância } \\
\text { explicada acumulada }\end{array}$ \\
\hline 1 & 2.9226 & 0.5374 \\
\hline 2 & 2.9246 & 0.5341 \\
\hline
\end{tabular}

Tabela 1. Teste Kaiser-Meyer-Olkin (KMO) e Doornik-Hansen para o protótipo 1 (30 itens) e protótipo 2 (22 itens) do instrumento sobre a combinação de alimentos para tornar o ferro mais biodisponível na dieta infantil. Rio de Janeiro (RJ), março de 2013 a junho de 2015.

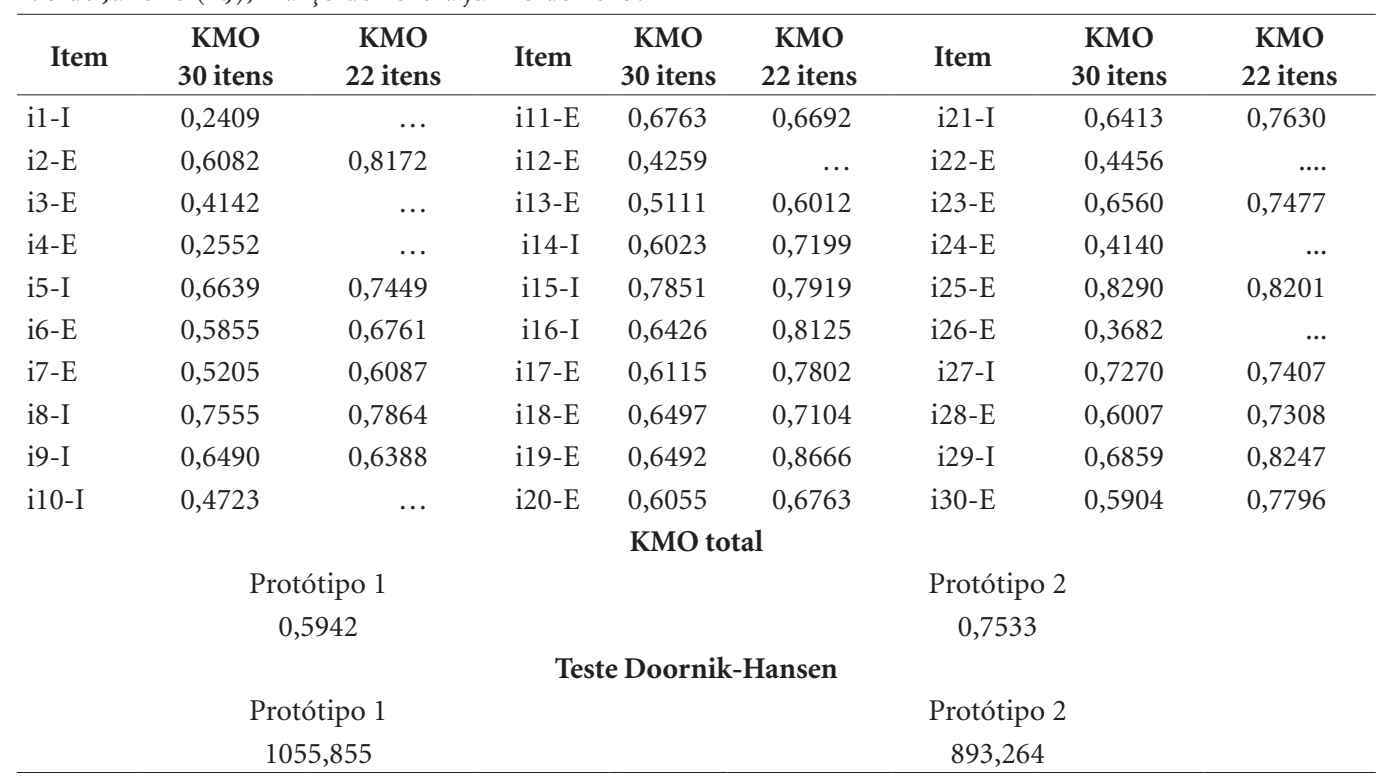

E: Estimuladores da absorção do ferro; I: Inibidores da absorção do ferro. (...) itens retirados, $\mathrm{KMO}<0,50$. 
que a estrutura do questionário, em relação à distribuição dos itens, deveria ser melhor avaliada.

Ao se estudar a estrutura fatorial do Protótipo 2, os itens inferidos como satisfatórios (cargas fatoriais > 0,40) se distribuíram em dois fatores: O Fator 1 abrigou os itens i2-E, i11-E, i17-E, i18-E, i19-E, i23E e i28E (combinações de alimentos que favorecem a absorção de ferro), com cargas fatoriais $(\lambda)$ variando de 0,4125 a 0,7235 e variância do erro $(\delta)$ de 0,4958 a 0,7587 . Analogamente, o Fator 2, os itens i5-I, i8-I, i9-I, i14-I, i15-I, i16-I, i21-I, i25-E e i29-I (combinações de alimentos que inibem a absorção de ferro, com exceção do i25-E), com cargas fatoriais $(\lambda)$ variando de 0,4256 a 0,7735 e variância do erro $(\delta)$ de 0,4680 a 0,8000. Ao Fator 1, denominou-se dimensão dos estimuladores da absorção de ferro na dieta e ao Fator 2, inibidores da absorção de ferro na dieta. Os itens i6-E, i7-E, i13-E, i20-E e i30-E foram retirados do questionário por terem apresentado cargas fatoriais baixas $(\lambda<0,40)$ e variâncias do erro expressivas. Em sua maioria, os itens apresentam variâncias do erro maiores do que 0,50, à exceção de i15-I, i23-E e i25-E. Os itens i27-I e i29-I apresentaram carga fatorial cruzada e variância do erro alta (Tabela 3), o que recomendou a retirada do questionário.

A correlação entre os fatores apresentou-se negativa $(-0,5313)$, sugerindo também a existência de dois fatores para o construto. O questionário foi reduzido a 15 itens, sendo sete itens do Fator 1 e oito itens do Fator 2, construindo-se o Protótipo 3 (Tabela 3).

Aplicando-se a rotação Promax ao Protótipo 3 com 15 itens, os autovalores (eigenvalue) para o Fator 1 alcançaram 2,9226 e para o Fator 2, o valor de 2,9246, com proporção da variância explicada acumulada de 0,5374 e 0,5341, respectivamente (Tabela 2). Esses achados permitem aquilatar maior parcimônia do modelo.

A análise das cargas fatoriais auferiu como satisfatórios, no Fator 1, os itens i2-E, i11-E, i17-E, i18-E, i19-E, i23E e i28E e, no Fator 2, os itens i5-I, i8-I, i9-I, i14-I, i15-I, i16-I, i21-I, i25-E. Conjugando os achados da Tabela 2 (autovalores e proporção da variância explicada acumulada) com os da Tabela 3 (mapeamento das cargas fatoriais), ratifica-se a bidimensionalidade do instrumento. O panorama das cargas fatoriais $(\lambda) \mathrm{e}$ das variâncias do erro $(\delta)$, entre os protótipos 2 e 3, apresentou pouca diferença, mas este modelo não apresentou cargas cruzadas, mostrando-se mais parcimonioso. A correlação entre os fatores (F1 e F2 $=-0,6140)$ reafirma a bidimensionalidade do questionário (Tabela 3 ).
Sendo este estudo exploratório, com vista a outro confirmatório, tornou-se imperativo reformular a semântica do item i25-E, que com a escrita na forma negativa (não beber..... não prejudicar....), pode ter induzido a respondente a entender o seu conteúdo como inibidor, uma vez que chá, mate e café são alimentos que tornam o ferro dietético menos biodisponível. Esse item, por causa de sua sintaxe, levou a matriz dos escores atribuídos aos itens alocá-lo no Fator 2. Neste sentido, foi imperativo reescrevê-lo de forma afirmativa, seja "evitar chá, mate e café durante e logo após às refeições para favorecer a absorção do ferro", transitando-o para o Fator 1, lócus pertinente ao grupo de estimuladores do ferro na dieta.

A partir dos achados, o questionário alcança a feição final do estudo exploratório (Quadro 2).

Avaliando-se a consistência interna do questionário, o Protótipo 3 (15 itens) em simulação, o coeficiente de Conbrach foi de 0,7725 (95\% IC $=0,7060$ a 0,8389$)$ para a dimensão alimentos estimuladores da absorção do ferro no Fator 1, com oito itens e de 0,7649 (95\% IC =0,7034 a 0,8263 ) para a dimensão alimentos inibidores da absorção do ferro no Fator 2, com sete itens. Em relação ao total do questionário, o valor foi de 0,8018 (95\% IC $=0,7429$ a 0,8606).

Os valores para a confiabilidade composta, a validade convergente e a validade discriminante apresentaram ligeira melhora (Tabela 3), do Protótipo 3 em comparação aos do Protótipo 2. No entanto, a modelagem não alcançou valores que indiquem que prevaleceu a quantidade de variância capturada pelos fatores sobre a quantidade de variância devido às medições de erros aleatórios (VME), o que indica reduzida captação da variável latente, pela não convergência dos escores dados pelas respondentes aos itens. A validade fatorial discriminante (raiz quadrada da VME) apresentou-se, nos dois fatores em ambos os protótipos, maior que a correlação entre os fatores, mostrando que os itens discriminam os conteúdos dos dois diferentes fatores.

\section{Discussão}

Pasquali ${ }^{18,23}$ sugeriu que, em estudos psicométricos, seriam necessários entre 200 e 300 sujeitos em estudo de mensuração comportamental. Assim também, na visão de Tabachnick e Fidell ${ }^{24}$, com menos de 300 sujeitos, uma análise fatorial ficaria comprometida. No presente estudo, o teste de KMO, aplicado à matriz dos escores observa- 
Tabela 3. Análise Fatorial Exploratória (AFE), Protótipo 2 e 3, do instrumento sobre a combinação de alimentos para tornar o ferro mais biodisponível na dieta infantil. Rio de Janeiro (RJ), março de 2013 a junho de 2015.

\begin{tabular}{|c|c|c|c|c|c|c|c|}
\hline \multirow{3}{*}{ item } & \multicolumn{3}{|c|}{ Protótipo 2} & \multicolumn{4}{|c|}{ Protótipo 3} \\
\hline & \multicolumn{3}{|c|}{$\begin{array}{c}\text { Modelo bidimensional }^{*} \\
\text { Sem constrangimento/Sem rotação }\end{array}$} & \multicolumn{4}{|c|}{$\begin{array}{l}\text { Modelo bidimensional }{ }^{\star *} \\
\text { Constrangido a dois fatores/Rotação }\end{array}$} \\
\hline & Fator 1 & Fator 2 & $\delta$ & & Fator 1 & Fator 2 & $\delta$ \\
\hline $\mathrm{i} 2-\mathrm{E}$ & 0,5576 & $-0,0292$ & 0,6992 & & 0,5425 & $-0,0076$ & 0,7078 \\
\hline i5-I & 0,0385 & 0,4896 & 0,7461 & & 0,0483 & 0,4745 & 0,7607 \\
\hline i6-E & 0,3693 & 0,0535 & 0,8475 & & & & \\
\hline iI7-E & 0,3449 & $-0,0267$ & 0,8865 & & & & \\
\hline i8-I & $-0,0932$ & 0,6229 & 0,6422 & & $-0,0778$ & 0,6089 & 0,6477 \\
\hline I9-I & $-0,0476$ & 0,4609 & 0,8000 & & $-0,0162$ & 0,4754 & 0,7777 \\
\hline i11-E & 0,5111 & $-0,0744$ & 0,7587 & & 0,4930 & $-0,0553$ & 0,7679 \\
\hline i13-E & 0,0999 & 0,1679 & 0,9506 & & & & \\
\hline i14-I & $-0,1413$ & 0,4835 & 0,7920 & & $-0,1243$ & 0,4792 & 0,7857 \\
\hline i15-I & $-0,2325$ & 0,7735 & 0,4680 & & $-0,1766$ & 0,7648 & 0,4535 \\
\hline i16-I & 0,1636 & 0,5844 & 0,5677 & & 0,2102 & 0,5776 & 0,5596 \\
\hline i17-E & 0,4125 & 0,1514 & 0,7651 & & 0,4717 & 0,1408 & 0,7235 \\
\hline i18-E & 0,6406 & 0,0145 & 0,5831 & & 0,6780 & 0,0263 & 0,5305 \\
\hline i19-E & 0,5746 & 0,0172 & 0,6629 & & 0,5822 & 0,0222 & 0,6538 \\
\hline i20-E & 0,3954 & $-0,0477$ & 0,8541 & & & & \\
\hline i21-I & $-0,0200$ & 0,6465 & 0,5903 & & 0,0470 & 0,6312 & 0,5841 \\
\hline $\mathrm{i} 23 \mathrm{E}$ & 0,7235 & $-0,0439$ & 0,4958 & & 0,7146 & $-0,0214$ & 0,4968 \\
\hline i25-I & 0,2245 & 0,5998 & 0,4996 & & 0,2393 & 0,5774 & 0,5381 \\
\hline i27-I & 0,2060 & 0,2530 & 0,8587 & & & & \\
\hline i28-E & 0,7193 & $-0,1085$ & 0,5231 & & 0,6970 & $-0,0812$ & 0,5368 \\
\hline i29-I & 0,3376 & 0,4256 & 0,6087 & & & & \\
\hline i30-E & 0,3484 & 0,0792 & 0,8538 & & & & \\
\hline Total & 6,1327 & 5,4930 & 15,4537 & & & & \\
\hline $\mathrm{CC}$ & 0,2841 & 0,2622 & & $\mathrm{CC}$ & 0,3125 & 0,3263 & \\
\hline VME & 0,2788 & 0,2497 & & VME & 0,2886 & 0,3075 & \\
\hline \multicolumn{4}{|c|}{ Raiz VME } & \multicolumn{4}{|c|}{ Raiz VME } \\
\hline & 0,5280 & 0,4997 & & & 0,5372 & 0,5545 & \\
\hline \multicolumn{4}{|c|}{ Correlação entre fatores } & \multicolumn{4}{|c|}{ Correlação entre fatores } \\
\hline & F1 & F2 & & & $\mathrm{F} 1$ & $\mathrm{~F} 2$ & \\
\hline $\mathrm{F} 1$ & 0,8472 & 0,7843 & & F1 & 0,7893 & 0,7968 & \\
\hline F2 & $-0,5313$ & 0,6204 & & F2 & $-0,6140$ & 0,6043 & \\
\hline
\end{tabular}

dos, em um questionário com 30 itens (Protótipo 1), em 151 mulheres, indicou que oito itens não alcançaram valor de KMO do maior que 0,50, como pressuposto de adequação amostral. Ao serem retirados esses itens (Protótipo 2, com 22 itens), o cenário, em relação ao KMO, mostrou que todos os itens se apresentaram adequados à análise fatorial. No que tange ao KMO total, seu valor teve um aumento expressivo. Acresce-se a esses achados que o teste de Doornik-Hansen, para ambos os Protótipos, apresentou norma- lidade multivariada. Os achados do presente estudo vêm ao encontro dessas recomendações científicas, mostrando a utilidade do KMO como critério para identificar se o modelo de análise fatorial, o qual foi utilizado, é adequadamente ajustado aos dados, testando a consistência geral dos dados. O KMO resulta em um índice, sendo uma estatística que indica a proporção da variância dos dados que pode ser considerada comum a todas as variáveis, ou seja, que pode ser atribuída a um fator comum. 
Quadro 2.Protótipo 3 (15 itens) do instrumento sobre a combinação de alimentos para tornar o ferro mais biodisponível na dieta infantil. Rio de Janeiro (RJ), março de 2013 a junho de 2015.

\begin{tabular}{|l|l|}
\hline Item & \multicolumn{1}{|c|}{ Protótipo 3 } \\
\hline i2-E & $\begin{array}{l}\text { Sempre dar na sobremesa uma fruta, pois contém vitamina C que ajuda na absorção do ferro no } \\
\text { organismo. }\end{array}$ \\
\hline i5-I & $\begin{array}{l}\text { Não consumir mate gelado no almoço e jantar por conter substâncias que dificultam a absorção do } \\
\text { ferro dos alimentos. }\end{array}$ \\
\hline i8-I & $\begin{array}{l}\text { Não comer iogurte após o almoço e jantar, pois o cálcio presente neste alimento compete com o ferro } \\
\text { contido nos legumes e verduras. }\end{array}$ \\
\hline i9-I & Não comer alimentos ricos em cálcio, como leite e queijos, antes nem após as grandes refeiçães. \\
\hline i11-E & $\begin{array}{l}\text { Comer os vegetais junto com a carne, pois o ferro contido na carne ajuda na absorção do ferro contido } \\
\text { nos vegetais, mas não se esquecer de comer frutas na sobremesa. }\end{array}$ \\
\hline i14-I & $\begin{array}{l}\text { Não colocar ovo no espinafre porque o espinafre contém substâncias que dificultam a absorção do ferro } \\
\text { do ovo. }\end{array}$ \\
\hline i15-I & $\begin{array}{l}\text { Não comer pudim de leite como sobremesa porque o cálcio presente atrapalha a absorção do ferro da } \\
\text { refeição. }\end{array}$ \\
\hline i16-I & Não beber café depois do almoço ou jantar, porque este irá atrapalhar a absorção do ferro. \\
\hline i17-E & Comer verduras verdes na dieta porque elas são ricas em ferro. \\
\hline i18-E & Comer frutas como sobremesa porque estas melhoram a absorção do ferro da dieta \\
\hline i19-E & $\begin{array}{l}\text { Comer carne, frango, peixe ou ovo duas vezes ao dia (almoço e jantar) com o objetivo de reduzir o risco } \\
\text { de anemia. }\end{array}$ \\
\hline i21-I & $\begin{array}{l}\text { Não beber leite ou derivados de leite como sobremesa no almoço e jantar para não prejudicar a } \\
\text { absorção do ferro. }\end{array}$ \\
\hline i23-E & Aumentar o consumo de frutas ricas em vitamina C para melhorar a absorção de ferro nas refeições. \\
\hline i25-E & Evitar chá mate e café durante e logo após às refeições para favorecer a absorção de ferro. \\
\hline i28-E & $\begin{array}{l}\text { Beber sucos de frutas cítricas como laranja, acerola e limão, pois, estes são ricos em vitamina C e } \\
\text { ajudam a melhorar a absorção do ferro do almoço e jantar. }\end{array}$ \\
\hline
\end{tabular}

No que tange à avaliação psicométrica do questionário, a comparação entre as cargas fatoriais dos protótipos 2 e 3 , mostra que o segundo protótico evoluiu pouco. No entanto, rotações oblíquas, ao relaxar a restrição de ortogonalidade, ganham simplicidade na interpretação, o que foi o caso, havendo redução do número de itens, de 22 para 15 . Abdi $^{25}$ lembra que o uso de rotações oblíquas foi fortemente recomendado por Thurstone ${ }^{26}$, mas são usadas mais raramente do que suas contrapartes ortogonais. Soluções oblíquas têm sido úteis na construção de teorias da Psicologia e Sociologia e provavelmente desempanham um papel relevante no desenvolvimento de teorias comportamentais. Osborne ${ }^{27}$ alertou que, para todas as rotações, o objetivo é “(...) simplificar e clarear as cargas fatoriais" e então a solução oblíqua irá produzir resultados cada vez mais claros. Assim, existe maior eficácia na utilização de “(...) rotações oblíquas para criar de forma clara padrões de resultados em AFE, onde os fatores são de fato correlacionados" 27 .

Pasquali ${ }^{23}$ revelou que cargas fatoriais altas em medidas comportamentais são difíceis de serem encontradas com dados observados, aceitando valores em torno de 0,30 . No entanto, Brown ${ }^{11}$, mais rigoroso, sugeriu cargas fatoriais acima de 0,40. A análise dos protótipos, do atual estudo, atendeu a segunda evidência. É importante alertar que cargas fatoriais altas não são de per si evidências de instrumentos parcimoniosos, ou seja, os itens selecionados devem guardar relação estreita com os pressupostos teóricos ${ }^{10,28}$ os quais, no presente estudo, fundamentam a biodisponibilidade de ferro em dietas ${ }^{5-7,29}$.

A estrutura bidimensional do questionário final (Protótipo 3), abrigando 15 itens, apresentou percentagem da variância explicada acumulada em torno de $50 \%$ da latência do construto para cada fator, permitindo inferir que o modelo se adere ao pressuposto teórico referente aos alimentos que estimulam e a outros que inibem a absorção do ferro dietético.

Embora as variâncias do erro não se apresentassem de forma tão parcimoniosa quanto o desejável $(0,5)$, o instrumento alcançou validade fatorial discriminante, o que indica que os itens apresentaram habilidade para discriminar as 
duas dimensões, estimuladores e inibidores da absorção de ferro na dieta.

No que tange ao seu potencial de aferição (confiabilidade), a disjunção entre o alfa de Cronbach e o coeficiente de confiabilidade composta corroboram a afirmativa dos autores Brown $^{11}$, Hair et al. ${ }^{12}$ e Raykov ${ }^{19}$, ou seja, que o alfa de Cronbach subestima a confiabilidade do instrumento que está sendo avaliado. Tais achados exigem estudos de replicação em grupos de interesse assemelhados.

São poucos os estudos que usam a análise fatorial para validar instrumentos no âmbito da dietética, mas estudos do comportamento alimentar vêm sendo realizados. Estudo fatorial exploratório de validação transcultural do Questionário do Comportamento Alimentar da Criança ${ }^{30}$, oriundo do Child Eating Behaviour Questionnaire - $\mathrm{CEBQ}^{31}$, com 249 mães portuguesas e respectivos filhos, utilizando análise de componentes principais com rotação Varimax, apresentaram cargas fatoriais para seis fatores entre 0,30 e 0,89 , tendo dois itens acusados: 0,12 e 0,13 . A percentagem de variância explicada para os seis fatores, do referido estudo, variou de $52,5 \%$ a $76,4 \%$ e, analogamente, os coeficientes de Conbrach (estimativa de ponto) variaram de 0,70 a 0,89 . Outro estudo sob o mesmo escopo foi realizado por Sleddens et al. ${ }^{32}$ com 135 pais de crianças holandesas, também utilizando análise de componente principal e rotação Varimax, apresentando, para sete fatores do instrumento, cargas fatoriais que variaram de 0,32 a 0,88 . A percentagem de variância explicada para os sete fatores variou de $42,00 \%$ a $70,00 \%$, e os coeficientes de Cronbach (estimativa de ponto) para os diferentes fatores do instrumento variaram de 0,72 a 0,91 . Estudo realizado por Real et al. ${ }^{33}$, testou uma versão combinada do Questionário de Alimentação Infantil e do conceito expandido do controle dos pais de forma explicitada e velada. A escala inicial apresentava 38 itens e foi respondida por 854 mães portuguesas. A AFE foi utilizada para avaliar a dimensionalidade do instrumento e a rotação Geomin foi aplicada para simplificar a estrutura das cargas fatoriais. O critério de autovalor indicou 11 fatores que explicaram 73,5\% do total da variância, e itens com cargas fatoriais iguais ou maiores do que 0,30 permaneceram no questionário. Em seguida, foi realizada a Análise Fatorial Confirmatória, agora com nove fatores e 32 itens, cujo alfa de Conbrach (estimativa de ponto) em relação aos fatores apresentou-se acima de 0,699. Comparando-se ao presente estudo, os estudos citados ${ }^{30,32}$, verificou-se que o tama- nho das amostras não diferiu expressivamente, à exceção do estudo de Real et al. ${ }^{33}$ No entanto, nesse último, os autores utilizaram a mesma amostra para realizar a AFE e, em seguida, a Análise Fatorial Confirmatória, o que não é recomendado ${ }^{10-12}$.

No que tange à estrutura fatorial, é importante ressaltar que os estudos de Viana e Sinde ${ }^{30} \mathrm{e}$ de Sleddens et al..$^{32}$ utilizaram análise de componente principal e rotação ortogonal Varimax. No entanto, Real et al. ${ }^{33}$ utilizaram a rotação oblíqua Geomin, que pressupõe correlação entre os fatores. O presente estudo utilizou a AFE para analisar o padrão de correlações existentes entre as variáveis (itens) e o referido padrão para agrupar as variáveis em fatores, ou seja, a partir de variáveis observadas inferir a latência do construto. Uma vez que Real et al..$^{33}$ pressupunham correlação entre os fatores (responsabilidade percebida pelos pais, peso dos pais percebido, peso da criança percebido, preocupação acerca do peso das crianças, restrições alimentares, pressão para comer, monitoramento da alimentação, controle explícito e controle velado do consumo alimentar), optaram por rotação oblíqua, similar ao presente estudo, tendo em vista que existe uma correlação inversa entre combinações de alimentos que favorecem a absorção de ferro e as que a inibem. A variação das cargas fatoriais, no presente estudo, pouco diferiu dos estudos realizados pelos autores, corroborando a observação de Pasquali ${ }^{23}$ sobre a dificuldade de se obter cargas fatoriais expressivas em medidas comportamentais.

A confiabilidade, expressa pelo coeficiente de Conbrach, com valores acima de 0,70, elegendo-se o limite inferior do intervalo de confiança apresentou-se bem próxima dos estudos cita$\operatorname{dos}^{30,33,34}$, lembrando que esses estudos apresentaram valores pontuais.

$\mathrm{Na}$ AFE, apesar da confiabilidade composta não satisfatória, a variância média estimada permitiu mostrar validade discriminante satisfatória. Um estudo subsequente, utilizando-se Análise Fatorial Confirmatória (AFC), poderá inferir sobre a adequabilidade da validade convergente, uma vez que o estudo exploratório eliminou itens com cargas cruzadas e alguns com variância do erro elevadas, e outros foram mantidos fundamentados em pressupostos teóricos. No presente estudo, a AFE foi usada para identificar itens potencialmente problemáticos que podem, consequentemente, causar ajustamento improdutivo na AFC em estudos posteriores ${ }^{21}$.

Para transpor a limitação do estudo, caberá a continuidade da validação do questionário por estudo confirmatório, o qual apresentará os ín- 
dices de ajuste destinados a verificar a qualidade do ajustamento. O modelo de AFC também possibilitará estruturas alternativas pela análise fatorial e melhores ajustes, com explicitação mais parcimoniosa, das correlações entre domínios ${ }^{34}$. Se houver bom ajuste do modelo de primeira ordem, um modelo de segunda ordem pode ser experimentado (Brown, 2006). O modelo final mostrará a invariância da estrutura fatorial, cargas fatoriais e fator variância-covariância através de grupos de interesse indicando as subescalas dos domínios ${ }^{35}$.

Modificações de itens, bem como criação de outros, podem ser requeridas no conjunto de itens como resultado de problemas metodológicos e conceituais, exigindo transculturalidade regional, tanto semântica quanto conjuntural. A possível falta de clareza conceitual dos itens $\mathrm{s}^{35}$ exigirá reescrita com ajustes semânticos para aplicação do instrumento a outros grupos de interesse.

Este estudo possui como contribuição o fato de ser o primeiro que cria um instrumento para avaliar a combinação de alimentos com o intuito de tornar o ferro mais biodisponível na dieta infantil, utiliza a análise fatorial e apresenta sua estrutura dimensional. É um dos poucos estudos que utiliza a confiabilidade composta em contraste com o alfa de Conbrach. As validades fatoriais, convergente e discriminante, pouco exploradas na literatura pertinente à construção de instrumento em Nutrição, sugerem adequada especificação dos domínios, ou seja, no presente estudo, Fator 1: estimuladores da absorção de ferro na dieta e Fator 2: inibidores da absorção de ferro na dieta, correspondendo ao que foi proposto teoricamente.
Nesta perspectiva, o questionário pode ser utilizado, mesmo necessitando de AFC, em futuros estudos da dietética contribuindo não só na produção de conhecimento científico mas também na abordagem para avaliação do consumo alimentar e orientação nutricional visando à prevenção da anemia ferropriva. Sua objetividade, por apresentar 15 itens, facilita sua aceitação por parte das pessoas convidadas a respondê-lo, segundo o compreendido por Selltiz et al..

Permite-se concluir que foi possível construir, em primeira feição, um instrumento validado para avaliar o conhecimento de mulheres adultas sobre a combinação de alimentos para tornar o ferro mais biodisponível na dieta infantil, adotando-se a análise fatorial como procedimento de análise de dados oriundos de respostas a uma escala Likert.

Acredita-se que o estudo exploratório veio suprir uma lacuna existente no campo da dietética e instigar ao prosseguimento de estudos ainda exploratórios e futuramente confirmatórios.

Embora o estudo tenha sido validado pelos procedimentos da AFE cabe ainda um estudo confirmatório utilizando-se a AFC ou Modelo de Equações Estruturais a fim de buscar a adequação do ajustamento por parâmetros que provem a habilidade do modelo, eleito neste estudo (Análise de Fator Principal), para reproduzir a relação observada entre os indicadores da matriz de entrada. É importante verificar a estabilidade de sua confiabilidade em grupos assemelhados e se o modelo de mensuração se generaliza. Este estudo pode ser visto como um caminho ao desenvolvimento de instrumentos de mensuração em saúde como subsídios para intervenção nutricional. 


\section{Colaboradores}

HS Lanzillotti e ME Barros: concepção, metodologia, análise de dados, na redação final e revisão crítica. FM Afonso e RMS Barbosa: planejamento e coordenação na pesquisa de campo e mineração de dados.

\section{Referências}

1. Brasil. Ministério da Saúde (MS). Oficina de trabalho "Carências Nutricionais: Desafio para Saúde Pública". Brasília: MS; 2004.

2. Jordão RE, Bernardi JLD, Barros Filho AA. Prevalência de anemia ferropriva no Brasil: uma revisão sistemática. Rev Paul Pediatr 2009; 27(1):90-98.

3. Rotemberg S, Vargas S. Práticas alimentares e o cuidado da saúde: da alimentação da criança à alimentação da família. Rev Bras Saúde Mater Infant 2004; 4(1):85-94.

4. Rodrigues LP, Jorge SRPF. Deficiência de ferro na mulher adulta. Revista Brasileira de Hematologia e Hemoterapia 2010; 32(Supl. 2):49-52.

5. Monsen ER, Balitfy JL. Calculating dietary iron bioavailability: refinement and computerization. JADA 1982; 80(4):307-311.

6. Cozzolino SF. Biodisponibilidade de nutrientes. Baruri: Manole; 2005.

7. Hallberg L, Hulthén L. Prediction of dietary iron absorption: an algorithm for calculating absorption and bioavailability of dietary iron. Am J Clin Nutr 2000; 71(5):1147-1160.

8. Benevides CMJ, Souza MV, Souza RDB, Lopes MV. Fatores antinutricionais em alimentos: revisão. Segur Aliment Nutr 2011; 18(2):67-79.

9. Selltiz C, Wrightsman LS, Cook SA. Métodos de pesquisa nas relações sociais. $2^{\mathrm{a}}$ ed. São Paulo: EPU; 1987.

10. Streiner DL, Norman GR. Health measurement scales: A practical guide to their development and use. $4^{\mathrm{a}} \mathrm{ed}$. Oxford: Oxford University Press; 2008.

11. Brown TA. Confirmatory Factor Analysis for Applied Research. New York, London: Guilford Press Methodology; 2006.

12. Hair JF, Black WC, Badin BJ, Anderson RE, Tatham RL. SEM: confirmatory factor analysis. $6^{\mathrm{a}}$ ed. Porto Alegre: Bookman; 2009.

13. Damasio BF. Uso da análise factorial exploratória em psicologia. Aval Psicol 2012; 11(2):213-228.

14. Field A. Discovering statistics using SPSS. In: Field A. Exploratory factor analysis. $4^{\mathrm{a}}$ ed. Thousand Oaks: Sage Publications; 2013. Chapter 17.

15. Stata Corp.. Stata multivariate Statistics Reference Manual release 12. College Station: A Stata Press Publication, Lakeway Drive; 2011.

16. Bonfim CB, Santos DN, Menezes IG, Reichenheim ME, Barreto ML. Um estudo sobre a validade de construto da Parent-Child Conflict Tactics Scale (CTSPC) em uma amostra populacional urbana do Nordeste brasileiro. Cad Saude Publica 2011; 27(11):2215-2226.

17. Hair JF, Black WC, Badin BJ, Anderson RE, Tatham RL. Análise Multivariada de dados. $6^{a}$ ed. Porto Alegre: Bookman; 2009.

18. Pasquali L. Instrumentos psicológicos: manual prático de elaboração. Brasília: Laboratório de Pesquisa em Avaliação e Medida (LabPAM). Instituto de Psicologia. Universidade de Brasília; 1999.

19. Raykov T. Scale Reliability, Cronbach's Coefficient Alpha, and violations of Essential Tau-Equivalence with Fixed Congeneric Components. Multivariate Behav Res 1997; 32(4):329-353. 
20. Kline RB. Principles and practice of structural equation modeling. New York: The Guilford Press; 2005.

21. Farrell AM, Rudd JM. Factor Analysis and Discriminant Validity: A Brief Review of Some Practical Issues. Adelaide: ANZMAC; 2009.

22. Maroco J, Oliveira RA, Vicente CS. Análise Fatorial do Inventário de Burnout de Maslach (mbi-hss) em Profissionais Portugueses. Psic Saúde \& Doenças 2013; 14(1):152-167.

23. Pasquali L. Análise fatorial para pesquisadores. Brasília: Laboratório de Pesquisa em Avaliação e Medida (LabPAM). Instituto de Psicologia. Universidade de Brasília; 2001.

24. Tabachnick B, Fidell LS. Using multivariate statistics. $4^{\mathrm{a}}$ ed. San Francisco: Allyn \& Bacon; 2001.

25. Abdi H. Factor Rotations in Factor Analyses. [accessed 2016 Jul 1]. Available from: http://www.utdallas. edu/ herve/Abdi-rotations-pretty.pdf

26. Thurstone LL. Multiple factor analysis. Chicago: University of Chicago Press; 1947.

27. Osborne JW. What is Rotating in Exploratory Factor Analysis? PARE 2015; 20(2):1-7.

28. Reichenheim ME, Moraes CL. Qualidade dos instrumentos epidemiológicos. In: Almeida-Filho N, Barreto M, organizadores. Epidemiologia \& Saúde. Fundamentos, Métodos e Aplicações. Rio de Janeiro: Guanabara -Koogan; 2011. p.150-164.

29. Monsen ER, Hallberget L, Layrisse M, Hegsted M, Cook JD, Mertz W, Finch CA. Estimation of available dietary iron. Am J Clin Nutr 1978; 31(1):134-141.

30. Viana V, Sinde S. O comportamento alimentar em crianças: Estudo de validação de um questionário numa amostra portuguesa (CEBQ). Análise Psicológica 2008; 1(26):111-120.
31. Wardle J, Guthrie C, Sanderson S, Rapoport L. Development of the Children's Eating Behaviour Questionnaire. J Child Psychol Psychiatry 2001; 42(7):963-970.

32. Sleddens EFC, Kremers SPJ, Thijs C. The Children's Eating Behaviour Questionnaire: factorial validity and association with Body Mass Index in Dutch children aged 6-7. IJBNPA 2008; 5:49.

33. Real H, Oliveira A, Severo M, Moreira P, Lopes C. Combination and adaptation of two tools to assess parental feeding practices in pre-school children. Eating Behaviors. 2014; 15(3):383-387.

34. Castro MMLD, Hokerberg YHM, Passos SRL. Validade dimensional do instrumento de qualidade de vida WHOQOL-BREF aplicado a trabalhadores da saúde. Cad Saude Publica 2013; 29(7):1357-1369.

35. Anderson CB, Hughes SO, Fisher JO, Nicklas TA. Cross-cultural equivalence of feeding beliefs and practices: The psychometric properties of child feeding questionnaire among Blacks and Hispanics. Prev Med 2005; 41(2):521-531.

Artigo apresentado em 29/03/2016

Aprovado em 22/11/2016

Versão final apresentada em 23/11/2016 